\title{
Coefficient estimates for a subclass of meromorphic bi-univalent functions defined by subordination
}

Ebrahim Analouei Adegani, Ahmad Motamednezhad and Serap Bulut

\begin{abstract}
In this work, we use the Faber polynomial expansion by a new method to find upper bounds for $\left|b_{n}\right|$ coefficients for meromorphic bi-univalent functions class $\Sigma^{\prime}$ which is defined by subordination. Further, we generalize and improve some of the previously published results.
\end{abstract}

Mathematics Subject Classification (2010): 30C45, 30C50.

Keywords: Coefficient estimates, Faber polynomial expansion, meromorphic functions, subordinate.

\section{Introduction and preliminaries}

Let $\mathcal{A}$ be a class of analytic functions in the open unit disk $\mathbb{U}=\{z \in \mathbb{C}:|z|<1\}$ of the form

$$
f(z)=z+\sum_{n=2}^{\infty} a_{n} z^{n}
$$

Also denote by $\mathcal{S}$ the class of all functions in $\mathcal{A}$ which are univalent and normalized by the conditions $f(0)=0=f^{\prime}(0)-1$. It is well known that every function $f \in \mathcal{S}$ has an inverse $f^{-1}$, which is defined by

$$
f^{-1}(f(z))=z \quad(z \in \mathbb{U}) \quad \text { and } \quad f\left(f^{-1}(w)\right)=w \quad\left(|w|<r_{0}(f) ; r_{0}(f) \geq \frac{1}{4}\right) .
$$

So, if $F$ is the inverse of a function $f \in \mathcal{S}$, then $F$ has the following representation

$$
F(w)=f^{-1}(w)=w+\sum_{n=2}^{\infty} \tilde{a}_{n} w^{n}
$$

which is valid in some neighborhood of the origin. 
In 1936, Robertson [23] introduced the concept of starlike functions of order $\alpha$ for $0 \leq \alpha<1$. A function $f \in \mathcal{A}$ is said to be starlike of order $\alpha$ if

$$
\Re\left(\frac{z f^{\prime}(z)}{f(z)}\right)>\alpha \quad(z \in \mathbb{U}) .
$$

This class is denoted by $\mathcal{S T}(\alpha)$. Note that $\mathcal{S T}(0)=\mathcal{S} \mathcal{T}$.

Definition 1.1. [8] For two functions $f$ and $g$ which are analytic in $\mathbb{U}$, we say that the function $f$ is subordinate to $g$ in $\mathbb{U}$, and write

$$
f(z) \prec g(z) \quad(z \in \mathbb{U}),
$$

if there exists a Schwarz function $\omega$, which is analytic in $\mathbb{U}$ with

$$
\omega(0)=0 \quad \text { and } \quad|\omega(z)|<1 \quad(z \in \mathbb{U}),
$$

such that

$$
f(z)=g(\omega(z)) \quad(z \in \mathbb{U}) .
$$

In particular, if the function $g$ is univalent in $\mathbb{U}$, then $f \prec g$ if and only if $f(0)=g(0)$ and $f(\mathbb{U}) \subseteq g(\mathbb{U})$.

Ma and Minda [20] have given a unified treatment of various subclass consisting of starlike functions by replacing the superordinate function $q(z)=\frac{1+z}{1-z}$ by a more general analytic function. For this purpose, they considered an analytic function $\varphi$ with positive real part on $\mathbb{U}$, satisfying $\varphi(0)=1, \varphi^{\prime}(0)>0$ and $\varphi$ maps the unit disk $\mathbb{U}$ onto a region starlike with respect to 1 , symmetric with respect to the real axis. The class $\mathcal{S T}(\varphi)$ of Ma-Minda starlike functions consists of functions $f \in \mathcal{S}$ satisfying

$$
\frac{z f^{\prime}(z)}{f(z)} \prec \varphi(z), \quad \text { for } z \in \mathbb{U} .
$$

It is clear that for special choices of $\varphi$, this class envelop several well-known subclasses of univalent function as special cases. The idea of subordination was used for defining many of classes of functions studied in the Geometric Function Theory, for example see $[7,21]$.

Let $\Sigma^{\prime}$ denote the class of meromorphic univalent functions $g$ defined in $\Delta:=$ $\{z \in \mathbb{C}: 1<|z|<\infty\}$ of the form

$$
g(z)=z+\sum_{n=0}^{\infty} \frac{b_{n}}{z^{n}} .
$$

Since $g \in \Sigma^{\prime}$ is univalent, it has an inverse $g^{-1}=G$ that satisfy

$$
g^{-1}(g(z))=z \quad(z \in \Delta) \quad \text { and } \quad g\left(g^{-1}(w)\right)=w \quad(M<|w|<\infty, M>0) .
$$

Furthermore, the inverse function $g^{-1}=G$ has a series expansion of the form

$$
G(w)=g^{-1}(w)=w+\sum_{n=0}^{\infty} \frac{\tilde{b}_{n}}{w^{n}} \quad(M<|w|<\infty) .
$$

A simple calculation shows that the inverse function $g^{-1}=G$, is given by

$$
G(w)=g^{-1}(w)=w-b_{0}-\frac{b_{1}}{w}-\frac{b_{2}+b_{0} b_{1}}{w^{2}}+\cdots .
$$


Let $(\mathcal{S T})^{\prime}(\varphi)$ denote the class of functions $g \in \Sigma^{\prime}$ which satisfy

$$
\frac{1}{z} \frac{g^{\prime}(1 / z)}{g(1 / z)} \prec \varphi(z), \quad \text { for } z \in \mathbb{U} .
$$

The mapping $f(z) \mapsto g(z):=1 / f(1 / z)$ establishes a one-to-one correspondence between functions in the classes $\mathcal{S}$ and $\Sigma^{\prime}$ and also between functions in the classes $\mathcal{S T}(\varphi)$ and $(\mathcal{S T})^{\prime}(\varphi)$ because (see for more details [5])

$$
\frac{z g^{\prime}(z)}{g(z)}=\frac{z(1 / f(1 / z))^{\prime}}{1 / f(1 / z)}=\frac{1}{z} \frac{f^{\prime}(1 / z)}{f(1 / z)}, \text { for }|z|>1 \text {. }
$$

Noth that if $g \in(\mathcal{S T})^{\prime}(\varphi)$, then there exists a unique function $f \in \mathcal{S} \mathcal{T}(\varphi)$ such that $g(z)=1 / f(1 / z)$. Also, it can be easily verified that $G(w)=1 / F(1 / w)$, where $F(w)$ is the inverse of $f(z)$.

Analogous to the bi-univalent analytic functions, a function $g \in \Sigma^{\prime}$ is said to be meromorphic bi-univalent if $g^{-1} \in \Sigma^{\prime}$. Examples of the meromorphic bi-univalent functions are

$$
z+\frac{1}{z}, \quad z-1, \quad-\frac{1}{\log \left(1-\frac{1}{z}\right)} .
$$

Determination of the sharp coefficient estimates of inverse functions in various subclasses of the class of analytic and univalent functions is an interesting problem in geometric function theory. Schiffer [24] obtained the estimate $\left|b_{2}\right| \leq \frac{2}{3}$ for meromorphic univalent functions $g \in \Sigma^{\prime}$ with $b_{0}=0$ and Duren [8] gave an elementary proof of the inequality $\left|b_{n}\right| \leq \frac{2}{n+1}$ on the coefficient of meromorphic univalent functions $g \in \Sigma^{\prime}$ with $b_{k}=0$ for $1 \leq k<\frac{n}{2}$. But the interest on coefficient estimates of the meromorphic univalent functions keep on by many researchers, see for example, [18, 19, 25, 26]. Several authors by using Faber polynomial expansions obtained coefficient estimates $\left|a_{n}\right|$ for classes meromorphic bi-univalent functions and bi-univalent functions, see for example $[10,12,13,14,15,16,17,28,27]$. First we recall some definitions and lemmas that used in this work.

Faber [9] introduced the Faber polynomials which play an important role in various areas of mathematical sciences, especially in geometric function theory. By using the Faber polynomial expansion of functions $g \in \Sigma^{\prime}$ of the form (1.3), the coefficients of its inverse map $g^{-1}=G$ defined in (1.5) may be expressed, (see for details [2] and [3]),

$$
G(w)=g^{-1}(w)=w-b_{0}-\sum_{n \geq 1} \frac{1}{n} K_{n+1}^{n} \frac{1}{w^{n}},
$$

where

$$
\begin{aligned}
K_{n+1}^{n}= & n b_{0}^{n-1} b_{1}+n(n-1) b_{0}^{n-2} b_{2}+\frac{n(n-1)(n-2)}{2} b_{0}^{n-3}\left(b_{3}+b_{1}^{2}\right) \\
& +\frac{n(n-1)(n-2)(n-3)}{3 !} b_{0}^{n-3}\left(b_{4}+3 b_{1} b_{2}\right)+\sum_{j \geq 5} b_{0}^{n-j} V_{j}
\end{aligned}
$$

such that $V_{j}$ with $5 \leq j \leq n$ is a homogeneous polynomial in the variables $b_{1}, b_{2}, \cdots, b_{n}$, (see for details [3]). 
60 Ebrahim Analouei Adegani, Ahmad Motamednezhad and Serap Bulut

Definition 1.2. [4] Let $\varphi$ is an analytic function with positive real part in the unit disk $\mathbb{U}$, satisfying $\varphi(0)=1, \varphi^{\prime}(0)>0, \varphi$ maps the unit disk $\mathbb{U}$ onto a region starlike with respect to 1 , symmetric with respect to the real axis. Such a function has series expansion of the form

$$
\varphi(z)=1+B_{1} z+B_{2} z^{2}+\cdots \quad\left(B_{1}>0\right) .
$$

Lemma 1.3. [8] Let $u(z)$ and $v(z)$ be two analytic functions in the unit disk $\mathbb{U}$ with

$$
u(0)=v(0)=0 \quad \text { and } \quad \max \{|u(z)|,|v(z)|\}<1 .
$$

We suppose also that

$$
u(z)=\sum_{n=1}^{\infty} p_{n} z^{n} \quad \text { and } \quad v(z)=\sum_{n=1}^{\infty} q_{n} z^{n} \quad(z \in \mathbb{U}) .
$$

Then

$$
\left|p_{1}\right| \leq 1, \quad\left|p_{2}\right| \leq 1-\left|p_{1}\right|^{2}, \quad\left|q_{1}\right| \leq 1, \quad\left|q_{2}\right| \leq 1-\left|q_{1}\right|^{2} .
$$

Lemma 1.4. $[1,2]$ Let the function $f \in \mathcal{A}$ be given by (1.1). Then for any $p \in \mathbb{Z}$, there are the polynomials $K_{n}^{p}$, such that

$$
\left(1+a_{2} z+a_{3} z^{2}+\cdots+a_{k} z^{k-1}+\cdots\right)^{p}=1+\sum_{n=1}^{\infty} K_{n}^{p}\left(a_{2}, a_{3}, \cdots, a_{n+1}\right) z^{n},
$$

where

$$
K_{n}^{p}\left(a_{2}, \cdots, a_{n+1}\right)=p a_{n+1}+\frac{p(p-1)}{2} D_{n}^{2}+\frac{p !}{(p-3) ! 3 !} D_{n}^{3}+\cdots+\frac{p !}{(p-n) !(n) !} D_{n}^{n},
$$

and

$$
D_{n}^{m}\left(a_{2}, a_{3}, \cdots, a_{n}\right)=\sum_{n=2}^{\infty} \frac{m !\left(a_{2}\right)^{\mu_{1}} \cdots\left(a_{n}\right)^{\mu_{n}}}{\mu_{1} ! \cdots \mu_{n} !}, \text { for } m \in \mathbb{N}=\{1,2, \ldots\} \text { and } m \leq n,
$$

the sum is taken over all nonnegative integers $\mu_{1}, \ldots, \mu_{n}$ satisfying

$$
\left\{\begin{array}{l}
\mu_{1}+\mu_{2}+\cdots+\mu_{n}=m \\
\mu_{1}+2 \mu_{2}+\cdots+n \mu_{n}=n
\end{array}\right.
$$

It is clear that $D_{n}^{n}\left(a_{2}, a_{3}, \cdots, a_{n}\right)=a_{2}^{n}$. In particular,

$$
\begin{aligned}
& K_{n}^{1}=a_{n+1}, \quad K_{1}^{2}=2 a_{2}, \quad K_{2}^{2}=2 a_{3}+a_{2}^{2}, \\
& K_{3}^{2}=2 a_{4}+2 a_{2} a_{3}, \quad K_{4}^{2}=2 a_{5}+2 a_{2} a_{4}+a_{3}^{2} .
\end{aligned}
$$

Lemma 1.5. [2, 3] and [6, page 52] Let the function $g \in \Sigma^{\prime}$ be given by (1.3). Then we have the following expansion

$$
\frac{z g^{\prime}(z)}{g(z)}=1+\sum_{n=0}^{\infty} F_{n+1}\left(b_{0}, b_{1}, \cdots b_{n}\right) \frac{1}{z^{n+1}}
$$

where

$$
F_{n+1}\left(b_{0}, b_{1}, \cdots b_{n}\right)=\sum_{i_{1}+2 i_{2}+\cdots+(n+1) i_{n+1}=n+1} A\left(i_{1}, i_{2}, \cdots, i_{n+1}\right)\left(b_{0}^{i_{1}} b_{1}^{i_{2}} \cdots b_{n}^{i_{n+1}}\right),
$$


and

$$
A\left(i_{1}, i_{2}, \cdots, i_{n+1}\right):=(-1)^{(n+1)+2 i_{1}+\cdots+(n+2) i_{n+1}} \frac{\left(i_{1}+i_{2}+\cdots+i_{n+1}-1\right) !(n+1)}{i_{1} ! i_{2} ! \cdots i_{n+1} !} .
$$

The first four terms of the Faber polynomials $F_{n}$ are given by

$$
\begin{aligned}
& F_{1}=-b_{0}, \quad F_{2}=b_{0}^{2}-2 b_{1}, \quad F_{3}=-b_{0}^{3}+3 b_{1} b_{0}-3 b_{2}, \\
& F_{4}=b_{0}^{4}-4 b_{0}^{2} b_{1}+4 b_{0} b_{2}+2 b_{1}^{2}-4 b_{3} .
\end{aligned}
$$

In this work, by using the Faber polynomial expansion we find upper bounds for $\left|b_{n}\right|$ coefficients by a new method for meromorphic bi-univalent functions class $\Sigma^{\prime}$ which is defined by subordination. Further, we generalize and improve some of the previously published results.

\section{Main results}

In this section, first we obtain estimates of coefficients $\left|b_{n}\right|$ of meromorphic biunivalent functions in the class $(\mathcal{S T})^{\prime}(\varphi)$. Next we obtain an improvement of the bounds $\left|b_{0}\right|$ and $\left|b_{1}\right|$ for special choices of $\varphi$.

Theorem 2.1. Let the function $g$ given by (1.3) and its inverse map $g^{-1}=G$ given by $(1.4)$ be in the class $(\mathcal{S T})^{\prime}(\varphi)$, where $\varphi$ is given by Definition 1.2 . If $b_{k}=0$ for $0 \leq k \leq n-1$, then

$$
\left|b_{n}\right| \leq \frac{B_{1}}{n+1} .
$$

Proof. From $g \in(\mathcal{S} \mathcal{T})^{\prime}(\varphi)$, we obtain

$$
\frac{1}{z} \frac{g^{\prime}(1 / z)}{g(1 / z)}=\frac{1-b_{1} z^{2}-2 b_{2} z^{3}-\cdots}{1+b_{0} z+b_{1} z^{2}+\cdots}=1-b_{0} z+\left(b_{0}^{2}-2 b_{1}\right) z^{2}+\cdots .
$$

Similar to Lemma 1.5, for function $g \in(\mathcal{S T})^{\prime}(\varphi)$ and for its inverse map $g^{-1}=G$, we have

$$
\begin{gathered}
\frac{1}{z} \frac{g^{\prime}(1 / z)}{g(1 / z)}=1+\sum_{n=0}^{\infty} F_{n+1}\left(b_{0}, b_{1}, \cdots b_{n}\right) z^{n+1}, \\
\frac{1}{w} \frac{G^{\prime}(1 / w)}{G(1 / w)}=1+\sum_{n=0}^{\infty} F_{n+1}\left(\tilde{b}_{0}, \tilde{b}_{1}, \cdots \tilde{b}_{n}\right) w^{n+1},
\end{gathered}
$$

respectively, where $\tilde{b}_{0}=-b_{0}, \tilde{b}_{n}=\frac{1}{n} K_{n+1}^{n}$.

On the other hand, since $g, G \in(\mathcal{S T})^{\prime}(\varphi)$, by the Definition 1.1, there exist two Schwarz functions $u, v: \mathbb{U} \rightarrow \mathbb{U}$ where $u, v$ are given by (1.8), so that

$$
\frac{1}{z} \frac{g^{\prime}(1 / z)}{g(1 / z)}=\varphi(u(z))=1+\sum_{n=1}^{\infty} \sum_{k=1}^{n} B_{k} D_{n}^{k}\left(p_{1}, p_{2}, \cdots, p_{n}\right) z^{n}
$$

and

$$
\frac{1}{w} \frac{G^{\prime}(1 / w)}{G(1 / w)}=\varphi(v(w))=1+\sum_{n=1}^{\infty} \sum_{k=1}^{n} B_{k} D_{n}^{k}\left(q_{1}, q_{2}, \cdots, q_{n}\right) w^{n}
$$


Comparing the corresponding coefficients of (2.2) and (2.4), we get that

$$
F_{n+1}\left(b_{0}, b_{1}, \cdots b_{n}\right)=\sum_{k=1}^{n+1} B_{k} D_{n+1}^{k}\left(p_{1}, p_{2}, \cdots, p_{n+1}\right) .
$$

Similarly, by comparing the corresponding coefficients of (2.3) and (2.5), we get that

$$
F_{n+1}\left(\tilde{b}_{0}, \tilde{b}_{1}, \cdots \tilde{b}_{n}\right)=\sum_{k=1}^{n+1} B_{k} D_{n+1}^{k}\left(q_{1}, q_{2}, \cdots, q_{n+1}\right) .
$$

Note that $b_{k}=0$ for $0 \leq k \leq n-1$, yields $\tilde{b}_{n}=-b_{n}$ and hence from (2.6) and (2.7), respectively, we get

$$
-(n+1) b_{n}=B_{1} p_{n+1},
$$

and

$$
-[-(n+1)] b_{n}=B_{1} q_{n+1} .
$$

By solving either of the above two equations for $b_{n}$ and applying $\left|p_{n+1}\right| \leq 1,\left|q_{n+1}\right| \leq 1$, we obtain

$$
\left|b_{n}\right| \leq \frac{B_{1}}{n+1}
$$

this completes the proof.

Corollary 2.2. Let the function g given by (1.3) and its inverse map $g^{-1}=G$ given by $(1.4)$ be in the class $(\mathcal{S T})^{\prime}\left(\left(\frac{1+z}{1-z}\right)^{\alpha}\right)$. If $b_{k}=0$ for $0 \leq k \leq n-1$, then

$$
\left|b_{n}\right| \leq \frac{2 \alpha}{n+1} \quad(0<\alpha \leq 1) .
$$

Corollary 2.3. [13] Let the function g given by (1.3) and its inverse map $g^{-1}=G$ given by $(1.4)$ be in the class $(\mathcal{S T})^{\prime}\left(\frac{1+(1-2 \beta) z}{1-z}\right)$. If $b_{k}=0$ for $0 \leq k \leq n-1$, then

$$
\left|b_{n}\right| \leq \frac{2(1-\beta)}{n+1} \quad(0 \leq \beta<1) .
$$

Corollary 2.4. Let the function $f$ given by (1.1) and its inverse map $f^{-1}=F$ given by (1.2) be in the class $\mathcal{S T}(\varphi)$. If $a_{k}=0$ for $2 \leq k \leq n-1$, then

$$
\left|a_{n}\right| \leq \frac{B_{1}}{n-1}
$$

Proof. Setting $f(1 / z):=1 / g(z)$ and $F(1 / w)=1 / G(w)$ in Theorem 2.1 we obtain the result and this completes the proof.

Corollary 2.5. ([14, Theorem 2.1]) Let the function $f$ given by (1.1) and its inverse map $f^{-1}=F$ given by $(1.2)$ be in the class $\mathcal{S} \mathcal{T}\left(\frac{1+A z}{1+B z}\right)$, where $A$ and $B$ are real numbers so that $-1 \leq B<A \leq 1$. If $a_{k}=0$ for $2 \leq k \leq n-1$, then

$$
\left|a_{n}\right| \leq \frac{A-B}{n-1} \text {. }
$$


Theorem 2.6. Let the function $g$ given by (1.3) and its inverse map $g^{-1}=G$ given by $(1.4)$ be in the class $(\mathcal{S T})^{\prime}(\varphi)$, where $\varphi$ is given by Definition 1.2. Then

$$
\left|b_{0}\right| \leq \frac{B_{1} \sqrt{B_{1}}}{\sqrt{\left|B_{1}^{2}-B_{2}\right|+B_{1}}}
$$

and

$$
\left|b_{1}\right| \leq \frac{B_{1}}{2}
$$

Proof. The equations (2.6) and (2.7) for $n=0$ and $n=1$, respectively, imply

$$
\begin{aligned}
-b_{0} & =B_{1} p_{1}, \\
b_{0}^{2}-2 b_{1} & =B_{1} p_{2}+B_{2} p_{1}^{2}, \\
b_{0} & =B_{1} q_{1}, \\
b_{0}^{2}+2 b_{1} & =B_{1} q_{2}+B_{2} q_{1}^{2} .
\end{aligned}
$$

From (2.10) and (2.12), we have

$$
p_{1}=-q_{1}
$$

and

$$
2 b_{0}^{2}=B_{1}^{2}\left(p_{1}^{2}+q_{1}^{2}\right) .
$$

Also by adding (2.11) and (2.13), and considering (2.15) we have

$$
\begin{aligned}
2 b_{0}^{2} & =B_{1}\left(p_{2}+q_{2}\right)+B_{2}\left(p_{1}^{2}+q_{1}^{2}\right) \\
& =B_{1}\left(p_{2}+q_{2}\right)+\frac{2 B_{2} b_{0}^{2}}{B_{1}^{2}} .
\end{aligned}
$$

So we obtain

$$
b_{0}^{2}=\frac{B_{1}^{3}\left(p_{2}+q_{2}\right)}{2\left(B_{1}^{2}-B_{2}\right)} .
$$

By (1.9), (2.10), (2.14) and the above equality give

$$
\begin{aligned}
\left|b_{0}\right|^{2} & \leq \frac{B_{1}^{3}\left(1-\left|p_{1}\right|^{2}\right)}{\left|B_{1}^{2}-B_{2}\right|} \\
& \leq \frac{B_{1}^{3}}{\left|B_{1}^{2}-B_{2}\right|}\left(1-\frac{\left|b_{0}\right|^{2}}{B_{1}^{2}}\right) .
\end{aligned}
$$

Therefore we obtain

$$
\left|b_{0}\right|^{2} \leq \frac{B_{1}^{3}}{\left|B_{1}^{2}-B_{2}\right|+B_{1}},
$$

which is the desired estimate on the coefficient $\left|b_{0}\right|$ as asserted in (2.8).

On the other hand, by subtracting (2.13) from (2.11) and considering (2.14) we get

$$
-4 b_{1}=B_{1}\left(p_{2}-q_{2}\right) \text {. }
$$

Taking the absolute values and considering (1.9) we obtain the desired estimate on the coefficient $\left|b_{1}\right|$ as asserted in (2.9). This completes the proof. 
64 Ebrahim Analouei Adegani, Ahmad Motamednezhad and Serap Bulut

Theorem 2.7. Let the function $g$ given by (1.3) and its inverse map $g^{-1}=G$ given by $(1.4)$ be in the class $(\mathcal{S T})^{\prime}\left(\left(\frac{1+z}{1-z}\right)^{\alpha}\right)$. Then

$$
\left|b_{0}\right| \leq \frac{2 \alpha}{\sqrt{\alpha+1}}
$$

and

$$
\left|b_{1}\right| \leq \alpha
$$

Remark 2.8. Theorem 2.7 is an refinement of estimate for $\left|b_{0}\right|$ obtained by Panigrahi [22, Corollary 2.3 ]. Also, for $\left|b_{1}\right|$ if $\frac{1}{\sqrt{5}}<\alpha \leq 1$ and $\left|b_{0}\right|$, Theorem 2.7 is an refinement of estimates obtained by Halim et al. [11, Theorem 2 ].

Theorem 2.9. Let the function g given by (1.3) and its inverse map $g^{-1}=G$ given by $(1.4)$ be in the class $(\mathcal{S T})^{\prime}\left(\frac{1+(1-2 \beta) z}{1-z}\right)$. Then

$$
\left|b_{0}\right| \leq\left\{\begin{array}{cc}
\sqrt{2(1-\beta)} \quad, \quad 0 \leq \beta \leq \frac{1}{2} \\
\frac{\sqrt{2}(1-\beta)}{\sqrt{\beta}} \quad, \quad \frac{1}{2} \leq \beta<1
\end{array}\right.
$$

and

$$
\left|b_{1}\right| \leq 1-\beta \text {. }
$$

Remark 2.10. Theorem 2.9 is an improvement of the estimates obtained by Panigrahi [22, Corollary 3.3] and also obtained by Halim et al. [11, Theorem 1].

\section{References}

[1] Airault, H., Remarks on Faber polynomials, Int. Math. Forum, 3(2008), 449-456.

[2] Airault, H., Bouali A, Differential calculus on the Faber polynomials, Bull. Sci. Math., 130(2006), 179-222.

[3] Airault, H., Ren, J., An algebra of differential operators and generating functions on the set of univalent functions, Bull. Sci. Math., 126(2002), 343-367.

[4] Ali, R.M., Lee, S.K., Ravichandran, V., Subramaniam, S., Coefficient estimates for biunivalent Ma-Minda starlike and convex functions, Appl. Math. Lett., 25(2012), 344-351.

[5] Ali, M.F., Vasudevarao, A., On coefficient estimates of negative powers and inverse coefficients for certain starlike functions, Proc. Math. Sci., 127(2017), 449-462.

[6] Bouali, A., Faber polynomials, Cayley-Hamilton equation and Newton symmetric functions, Bull. Sci. Math., 130(2006), 49-70.

[7] Bulboacă, T., Differential Subordinations and Superordinations, Recent Results, House of Scientific Book Publ., Cluj-Napoca, 2005.

[8] Duren, P.L., Univalent Functions, Grundlehren der Mathematischen Wissenschaften, Band 259, Springer-Verlag, New York, Berlin, Heidelberg and Tokyo, 1983.

[9] Faber, G., Über polynomische Entwickelungen, Math. Ann., 57(1903), 389-408.

[10] Frasin, B.A., Aouf, M.K., New subclasses of bi-univalent functions, Appl. Math. Lett., 24(2011), 1569-1573. 
[11] Halim, S.A., Hamidi, S.G., Ravichandran, V., Coefficient estimates for meromorphic bi-univalent functions, arXiv:1108.4089v1 [math.CV](2011).

[12] Hamidi, S.G., Halim, S.A., Jahangiri, J.M., Coefficient estimates for a class of meromorphic bi-univalent functions, C.R. Math. Acad. Sci. Paris, 351(2013), 349-352.

[13] Hamidi, S.G., Halim, S.A., Jahangiri, J.M., Faber polynomial coefficient estimates for meromorphic bi-starlike functions, Int. J. Math. Math. Sci., 2013(2013), Art. ID 498159, $4 \mathrm{p}$.

[14] Hamidi, S.G., Jahangiri, J.M., Faber polynomial coefficients of bi-subordinate functions, C.R. Math. Acad. Sci. Paris, 354(2016), 365-370.

[15] Hamidi, S.G., Janani, T., Murugusundaramoorthy, G., Jahangiri, J.M., Coefficient estimates for certain classes of meromorphic bi-univalent functions, C.R. Math. Acad. Sci. Paris, 352(2014), 277-282.

[16] Jahangiri, J.M., Hamidi, S.G., Coefficient estimates for certain classes of bi-univalent functions, Int. J. Math. Math. Sci., 2013(2013), Art. ID 190560, 4p.

[17] Jahangiri, J.M., Hamidi, S.G., Halim, S.A., Coefficients of bi-univalent functions with positive real part derivatives, Bull. Malays. Math. Sci. Soc., 37(2014), 633-640.

[18] Kapoor, G.P., Mishra, A.K., Coeffcient estimates for inverses of starlike functions of positive order, J. Math. Anal. Appl., 329(2007), 922-934.

[19] Kubota, Y., Coeffcients of meromorphic univalent functions, Kodai Math. Sem. Rep., 28(1976/77), 253-261.

[20] Ma, W., Minda, D., A unified treatment of some special classes of univalent functions, in: Proceedings of the Conference on Complex Analysis, Z. Li, F. Ren, L. Lang and S. Zhang (eds), Int. Press, 1994, 157-169.

[21] Miller, S.S., Mocanu, P.T., Differential Subordinations: Theory and Applications, Series on Monographs and Textbooks in Pure and Appl. Math., vol. 255, Marcel Dekker, Inc., New York, 2000.

[22] Panigrahi, T., Coefficient bounds for certain subclasses of meromorphic and bi-univalent functions, Bull. Korean Math. Soc., 50(2013), 1531-1538.

[23] Robertson, M.I.S., On the theory of univalent functions, Ann. of Math., 37(1936), 374408.

[24] Schiffer, M., Sur un probléme dextrémum de la repré sentation conforme, Bull. Soc. Math. France, 66(1938), 48-55.

[25] Schober, G., Coeffcients of inverses of meromorphic univalent functions, Proc. Amer. Math. Soc., 67(1977), 111-116.

[26] Springer, G., The coeffcient problem for schlicht mappings of the exterior of the unit circle, Trans. Amer. Math. Soc., 70(1951), 421-450.

[27] Zireh, A., Analouei Adegani, E., Bidkham, B., Faber polynomial coefficient estimates for subclass of bi-univalent functions defined by quasi-subordinate, Math. Slovaca, 68(2018), 369-378.

[28] Zireh, A., Analouei Adegani, E., Bulut, S., Faber polynomial coefficient estimates for a comprehensive subclass of analytic bi-univalent functions defined by subordination, Bull. Belg. Math. Soc. Simon Stevin, 23(2016), 487-504. 
66 Ebrahim Analouei Adegani, Ahmad Motamednezhad and Serap Bulut

Ebrahim Analouei Adegani

Faculty of Mathematical Sciences,

Shahrood University of Technology

P.O.Box 316-36155, Shahrood, Iran

e-mail: analoey.ebrahim@gmail.com

Ahmad Motamednezhad

Faculty of Mathematical Sciences,

Shahrood University of Technology

P.O.Box 316-36155, Shahrood, Iran

e-mail: Corresponding author: a.motamedne@gmail.com

Serap Bulut

Kocaeli University,

Faculty of Aviation and Space Sciences

Arslanbey Campus,

41285 Kartepe-Kocaeli, Turkey

e-mail: serap.bulut@kocaeli.edu.tr 